\title{
Competencias Generales en Medicina, Rol de la Anatomía
}

\author{
Dr. Oscar Inzunza H.
}

En los albores del siglo XXI, las escuelas de Medicina del mundo entero se encuentran involucradas en un proceso de cambio curricular orientado a redefinir -en función de los progresos de la medicina, las nuevas estrategias docentes y de las demandas de la comunidad- los conocimientos, habilidades, actitudes y destrezas exigibles a los estudiantes al final de su periodo de formación médica de pregrado (Rolfe et al., 2002). Esta tendencia mundial, que ha sido llamada por algunos autores "el movimiento internacional por las competencias", ha permitido ir definiendo, en las distintas escuelas de Medicina, una serie de competencias generales (Dannefer \& Henson, 2007) para luego plantear y desarrollar competencias profesionales específicas para cada especialidad (Kilroy \& Driscoll, 2006, Edgren, 2006, Sanson-Fisher et al., 2005, Aravena \& GarcíaHernández, 2006). Para los morfólogos en general y para los anatomistas en particular, este diseño curricular presenta nuevos desafíos, toda vez que este proceso impactará profundamente en nuestro quehacer docente. Así, la definición consensuada -entre los distintos actores académicos, asistenciales, sociales, empresariales y políticos- del bagaje clínico y del nivel de desempeño exigible al médico recién formado, permitirá desarrollar un currículo médico acorde con las necesidades del país -o de la región; siendo este diseño suficientemente plástico como para readecuarlo en función de las nuevas prioridades que demande la realidad médica a futuro.

En este contexto, y en el marco de nuestro reciente IX Congreso de Anatomía del Cono Sur (Talca, 29 al 31 de octubre de 2007), resulta interesante analizar en esta prestigiosa tribuna, el Acta $\mathrm{N}^{\mathrm{o}} 1$ de la Comisión Consultiva de Expertos en Medicina del MERCOSUR, documento titulado "Dimensiones, componentes, criterios e indicadores para la acreditación MERCOSUR", que fue refrendado por los ministros de educación de los Miembros Plenos (Argentina, Paraguay, Uruguay y Brasil) y de los Estados Asociados (Bolivia y Chile) del pacto regional, en la ciudad de Buenos Aires, Argentina, en junio de 2002 (www.mec.gov.py/ aneaes/docs/Dimensiones\%20Medicina.pdf). En las páginas 6 a 8 del citado documento, bajo el punto 2.1.1.2-, se especifica un número de diez competencias generales que definen las capacidades deseables en un egresado de una Escuela de Medicina de la región (Tabla I).
Tabla I. Competencias del egresado, Comisión Consultiva de Expertos en Medicina, MERCOSUR/RME.- ACTA No 1/2002.

* Asumir una conducta ética frente al paciente, el equipo de salud y la comunidad.

* Conocer la realidad sanitaria de su medio.

* Valorar el uso de la anamnesis y el examen físico como elementos fundamentales de la práctica médica.

* Realizar procedimientos clínicos y quirúrgicos indispensables en la atención inicial de las urgencias y emergencias.

* Diagnosticar y tratar correctamente las principales enfermedades del ser humano en todas las fases de su desarrollo, teniendo en cuenta criterios de prevalencia y potencial mórbido.

* Reconocer y derivar y/o referir adecuada y oportunamente pacientes portadores de problemas, que están fuera del alcance de su competencia.

* Promover estilos de vida saludables, en los ámbitos individuales y colectivos.

* Desarrollar la capacidad, sensibilidad y pertinencia en la relación con el paciente, sus familiares y el equipo de salud.

* Considerar las relaciones de costo beneficio y riesgo benéfico en las decisiones de todos los procedimientos médicos.

* Utilizar metodología científica en la práctica profesional y actitud crítica en la lectura de la literatura médica.

De estas competencias quiero referirme a dos de ellas, que demandan aspectos psico-sociales (aptitudes y actitudes) esenciales en la formación del estudiante de Medicina, buscando los puntos donde la docencia anatómica puede y debe influir para la consecución de tales características en el aprendiz.

1.- "Desarrollar la capacidad, sensibilidad y pertenencia en la relación con el paciente".

Alguien podría refutar argumentando que los alumnos de primer año de la Carrera de Medicina no tratan pacientes y éticamente sería incorrecto que lo hicieran. Pero debemos considerar que el primer paciente que tiene un estudiante de Medicina es el cadáver utilizado en Anatomía. Metafóricamente, de él se nutre y aprende. Su primer encuentro con el cadáver le define claramente la realidad de la vida humana, lo proyecta a su objetivo final -el paciente vivoy lo confronta con la gran responsabilidad que está asumiendo al formarse como Médico (Granger, 2004). Para Pellegrino (1974), el encuentro del estudiante con el cadáver constituye el "punto nodal" en la educación médica que -con un 
adecuado manejo y fortalecimiento- conduce a la "objetividad compasiva", característica esencial del quehacer médico. De acuerdo con Engel (1971), en este proceso de "metamorfosis", el aprendiz no sólo "asimila algo nuevo, más bien se convierte en un ser nuevo". Esta actitud frente al que sufre constituye hoy en día un desafío, considerando la tecnificación creciente del trabajo médico y la focalización de la educación médica en los procedimientos clínicos.

La actividad práctica en Anatomía, utilizando el cadáver humano como centro, constituye la única oportunidad que tiene el Médico en ciernes para confirmar empíricamente la situación de los órganos y sistemas corporales y su relación con la enfermedad y la muerte. La clásica pregunta de nuestros alumnos "y de eso murió?", denota una reflexión acerca de la condición de vida de la persona, cuyo cuerpo ahora yace en la mesa de mármol y de cómo sobrellevó la afección que se revela en el noble acto de la disección. Entonces, la enfermedad deja de ser un concepto teórico y abstracto, por el contrario, se hace real y patente en el hígado nodular y fibroso de la cirrosis, en la úlcera indurada y los linfonodos celíacos voluminosos del cáncer gástrico, en la dilatación cardiaca de la insuficiencia aórtica, en la esplenomegalia del SIDA o en las escaras del anciano caquéctico; hallazgos constantes en nuestras disecciones y que todo anatomista debe señalar a sus alumnos.

En el contexto actual de promoción de donaciones de órganos y el creciente interés de la opinión pública en "donar su cuerpo para la ciencia", -hecho colocado en el tapete, con la misa organizada por docentes y alumnos de la Escuela de Medicina de la Universidad de Chile por el alma de sus cadáveres donados-, este estilo de aprendizaje centrado en el cadáver resalta el privilegio de ser el receptor de una voluntad postrera; alguien, desinteresadamente, se entrega al aprendiz para que éste se forme como Médico, un preciado presente que se materializa con la muerte del donante. Del mismo modo, el aprendiz así sensibilizado podrá aquilatar la confianza que en él depositará más tarde el paciente de sala común, para que lo examine y se entrene como médico. Esto aparece claramente expresado en el comentario de una alumna de Noelle A. Granger, de la Universidad de Carolina del Norte, en su acotación "El trabajo con cadáveres representó un elemento crítico en el desarrollo del sentimiento compasivo hacia mis futuros pacientes" (Granger).

Aún cuando mucho ha cambiado en el último tiempo la forma de diagnosticar, tratar y prevenir las enfermedades, todavía el tema central de la medicina sigue siendo la persona que sufre la enfermedad, el dolor y la muerte. El cadáver representa un eslabón con el paciente, -el que ahora es llamado por su connotación económica "cliente"-, y brinda el modo óptimo de entrenamiento en el diagnóstico para quien desea ofrecer tratamiento y curación. La exclusión o reducción del trabajo en el cadáver durante el proceso de formación médica se opone al entrenamiento de profesionales competentes, compasivos, comunicativos y con una visión centrada en el paciente. Sin duda, esto constituye un desafío enorme para nosotros los anatomistas quienes, como mentores, debemos atender y considerar los requerimientos cognitivos específicos del ramo, además de los elementos afectivos y emocionales que surgen en el devenir de cada paso práctico de Anatomía; todo esto en un período de tiempo cada vez más restringido.

2.- "Utilizar metodología científica en la práctica profesional y actitud crítica en la lectura de la literatura médica".

Respecto de la metodología científica, Andrés Vesalio, el año 1542, con su obra De humani corpori fabrica inaugura la era de la Medicina basada en la ciencia, al confrontar la información anatómica existente en la Edad Media, cuyo origen se remontaba a Galeno, con los hechos revelados mediante la disección en cadáveres humanos. El enfoque utilizado por Vesalio establece las etapas iniciales del "método científico, esto es: La recolección de datos, mediante la observación directa en el cadáver; la formulación de una explicación provisoria de los hechos observados, la hipótesis; y la contrastación de la hipótesis. Así, al colocar el cuerpo humano en el centro de su investigación, Vesalio implícitamente reafirma el canon Hipocrático de la Medicina basada en el paciente (Aziz et al., 2002). Más tarde, William Harvey complementa el paradigma Vesaliano, incorporando el análisis cuantitativo y la experimentación en modelos animales, como un refinamiento del método de diagnóstico basado en la evidencia. Entonces, comenzar el proceso de formación médica con un curso de Anatomía basado en la observación cadavérica, significa inculcar, desde muy temprano, el método científico en el aprendiz, método que es la base del diagnóstico clínico. En efecto, la descripción detallada de los signos y los síntomas observados en el paciente sigue siendo el proceso nuclear del análisis clínico que fundamenta la hipótesis diagnóstica. La exclusión o reducción del trabajo en el cadáver, durante la formación médica, restringe y menoscaba la posibilidad de desarrollar e internalizar en el estudiante este proceso de observación crítica de los hechos y la rigurosidad en la interpretación de ellos para arribar a un diagnóstico preciso.

Así como el ritmo de la práctica médica es: observación - historia - examen clínico - interpretación - hipótesis diagnóstica - plan de tratamiento, siendo el paciente el elemento central del proceso; el ritmo de la observación anatómica sigue una secuencia similar: observación - análisis comparativo - interpretación de los hallazgos - diagnóstico diferencial de las estructuras anatómicas - exploración para con- 
trastar las diferentes hipótesis sugeridas; siendo en este caso el cadáver el recurso central y capital del análisis. Entonces, un sólido conocimiento anatómico, forjado en el trabajo práctico con material cadavérico, constituye la mejor preparación para que el aprendiz logre un buen desempeño en el examen clínico de sus pacientes y en la práctica de procedimientos en forma eficiente y segura; habilidades y destrezas que se han visto seriamente debilitadas en los alumnos de aquellas escuelas de medicina que abandonaron el trabajo anatómico con material cadavérico, tal como ocurrió en la Universidad de California en San Francisco, en la Universidad de California en Davis y en la Universidad de Washington, según el reciente reporte de Rizzolo \& Stewart (2006). Una situación similar hemos detectado en nuestro medio, al analizar el rendimiento en pruebas de reconocimiento de estructuras anatómicas por parte de médicos de reciente graduación (Inzunza et al., 2007).

Respecto de la actitud crítica en la lectura de literatura científica, a pesar de la obvia significancia de la variabilidad humana, incluyendo las anomalías del desarrollo, la tendencia en el último cuarto de siglo ha sido la de presentar al cuerpo humano como un tipo idealizado, normal, de modo que los estudiantes aprenden la Anatomía de un prototipo prácticamente inexistente. En efecto, los antiguos textos de Anatomía presentaban anexos o notas a pie de pagina con detalles de las variaciones anatómicas y su frecuencia. Los textos modernos han prescindido de tan importante información. Recordemos aquí las palabras del profesor Liberato DiDio "la variación anatómica es el hallazgo más frecuente", o la afirmación que él cita de Castaldi "En el Homo Sapiens, no existe el hombre ideal, existen simplemente hombres" (DiDio, 1970). Esta sobresimplificación de la realidad anatómica, característica del posmodernismo, ha llevado a un empobrecimiento del entrenamiento médico, situación que ha redundado en diagnósticos erróneos y en mala praxis (Aziz et al.). Entonces, con el propósito de preparar al aprendiz ante la certeza impredecible de la variación anatómica, es necesario brindarle las posibilidades de que se entrene en la observación crítica del material cadavérico a fin de detectarlas y explicarlas, cuando corresponda, desde la Embriología. A este respecto, resulta motivador ver la cara de sorpresa de nuestros alumnos cuando se encuentran con una arteria lusoria, o un foramen oval permeable, o el origen aórtico de la vertebral izquierda; y más gratificante resulta ver la cara de satisfacción del aprendiz cuando ha encontrado en la embriología la explicación del fenómeno observado (Figueroa et al., 2007a y b). Esto se recoge claramente en el comentario de un alumno de Noelle A. Granger, de la Universidad de Carolina del Norte, en su acotación: "Para mi, una de las más importantes lecciones que aprendí en Anatomía fue el amplio rango de variabilidad anatómica, situaciones que nunca son causa de disfunción" (Granger).
Desde mi punto de vista, la expresión máxima de este "análisis crítico de la literatura" se materializa cuando mis alumnos de primer año son capaces de detectar los errores de nuestro texto guía "Clinically Oriented Anatomy" de KL Moore y AF Dalley, fourth edition, 1999, (que son pocos), o los defectos de la traducción de ese libro al español (que son muchos). Es decir, cuando ellos encuentran una situación irreconciliable entre la descripción de un respetable texto de Anatomía con lo que ellos observan en el cadáver. Si mis alumnos son capaces de realizar este proceso deductivo, yo me doy por satisfecho. En este ejercicio, los aprendices han logrado llegar a la verdad anatómica con el texto o contra el texto, con el docente o contra el docente; con fuerza la verdad anatómica se ha revelado en la observación crítica del material cadavérico. Esto augura una futura asertividad en sus diagnósticos.

Finalmente, recojo el comentario de otro alumno de Noelle A. Granger, de la Universidad de Carolina del Norte, esta vez un Médico graduado en el curso 2004, quien señala respecto del curso de Anatomía: "Es el único de los cursos de los primeros años de la Carrera que hace explícitos el privilegio y el desafío de ser Médico, porque sólo el laboratorio de Anatomía te enseña la intimidad del cuerpo humano" (Granger). Esta sentida aseveración debe hacernos reflexionar sobre la formación médica en nuestro medio, donde asistimos a una proliferación de Escuelas de Medicina, cuyos cursos de Anatomía son atendidos por profesionales que carecen de una formación y una trayectoria en los ámbitos de la Morfología, quienes, de manera acomodaticia, prefieren tratar estos importantes temas en modelos o software indefectiblemente inexactos y ficticios.

\section{REFERENCIAS BIBLIOGRÁFICAS}

Aravena V.V. \& García-Hernández F. Anatomía y competencias clínicas en odontología. Estudio basado en apreciación de académicos. Int. J. Morphol., 24(4):713-20, 2006.

Aziz M. A.; McKenzie J. C.; Wilson J. S.; Cowie R. J.; Ayeni S.A. \& Dunn B.K. The human cadaver in the age of biomedical informatics. Anat. Rec. (New Anat), 269:2032, 2002.

Dannefer E. F. \& Henson L. C. The portfolio approach to competency-based assessment at the Clevelant Clinic Lerner College of Medicine. Acad. Med., 82:493-502, 2007.

DiDio L. J. A. Sinopsis of Anatomy. Saint Louis, Mosby, 1970. 
Edgren, G. Developing a competence-based core curriculum in biomedical laboratory science: a Delphi study. Medical Teacher 28(5):409-17, 2006.

Engel G. L. Care and feeding of the medical student: The foundation for professional competence. JAMA 215:1135-141, 1971.

Figueroa C.; Prada C.; Rioseco F.; Vargas A. \& Inzunza O. Arteria subclavia aberrante. Int. J. Morphol., 25(1):159, 2007a.

Figueroa C.; Prada C.; Rioseco F.; Vargas A. \& Inzunza O. Arteria vertebral izquierda aberrante. Int. J. Morphol., 25(1):159, $2007 \mathrm{~b}$.

Granger N.A. Dissection laboratory is vital to medical gross anatomy education. Anat. Rec. (Part B: New Anat), 281B:6-8, 2004.

Inzunza O.; Vargas A. \& Bravo H. Anatomía y Neuroanatomía, disciplinas perjudicadas por la reforma curricular. Int. J. Morphol., 25(4):819-24, 2007.

Kilroy D. \& Driscoll P. Determination of required anatomical knowledge for clinical practice in emergency medicine: national curriculum planning using a modified Delphi technique. Emerg. Med. J., 23:693-6, 2006.

MERCOSUR/RME.- ACTA No 1/2002, www.mec.gov.py/ aneaes/docs/Dimensiones\%20Medicina.pdf

Pellegrino E. D. Educating the humanist physician: An ancient ideal reconsidered. JAMA, 227:1288-94, 1974.

Rizzolo L. J. \& Stewart W.B. Should we continue teaching anatomy by dissection when...? Anat. Rec. (Part B: New Anat)., 289B:215-8, 2006.

Rolfe. I. E .; Pearson S. A.; Sanson-Fisher R.W.; Ringland C.; Bayley S.; Hart H. \& Kelly S. Which common clinical condition should medical students be able to manage by graduation? A perspective from Australian interns. Medical Teacher., 24(1):16-22, 2002.

Sanson-Fisher R.W.; Rolfe I. E. \& Williams, N. Competency based teaching: the need for a new approach to teaching clinical skills in the undergraduate medical education course. Medical Teacher 27(1):29-36, 2005.
Dirección para correspondencia:

Prof. Dr. Oscar Inzunza

Departamento de Anatomía Normal

Escuela de Medicina,

Pontificia Universidad Católica de Chile

CHILE

Teléfono 56-2-354 3076

Email: oinzunza@med.puc.cl

Recibido : 31-01-2008

Aceptado: 07-03-2008 